\section{Detection of Elementary Sulphur}

A REACrion is known to take place when organic compounds containing hydrogen are heated above the melting point with elementary sulphur. Among other decomposition products hydrogen sulphide is evolved, and its evolution is especially marked when a polyhydric phenol, for example, hydroquinone or pyrogallol, is the reducing agent. The generation of hydrogen sulphide for laboratory use from a melted mixture of sulphur and paraffin wax is perhaps a more familiar example of the reaction. So far as can be ascertained from the literature, the analytical possibilities of the reaction have not been explored previously, yet it affords a means of detecting sulphur quickly and conveniently in complex or coloured materials, provided they are not too readily combustible.

A small quantity of the substance to be tested is mixed in the dry, powdered state with an equal weight of a polyhydric phenol, for example, hydroquinone or pyrogallol, and the mixture heated in a bulb tube to melting point.

A freshly moistened lead acetate paper held in the issuing vapours is promptly stained brown when sulphur is prasent. Under such simple conditions it has been found that 0.0001 gm. of sulphur can be detected, but it is possible that an even higher degree of sensitivity might be attained.

The behaviour of the test with a few typical

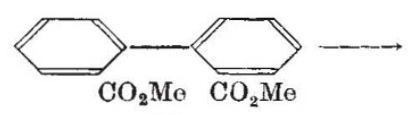

(v)

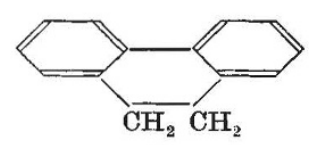

(I) carbon requires experimental procedure which is unattainable in many university laboratories. Burger and Mosettig1 effected the dihydrogenation by using a copper-chromium-barium oxide catalyst at $220^{\circ}$, with a hydrogen pressure of about 200 atmospheres over a period of about nine hours.

Wittig and his co-workers ${ }^{2}$ had observed the conversion of benzyl bromide into $s$-diphenylethane by the action of lithium phenyl, which produced lithium benzyl, the latter at once reacting with unchanged benzyl bromide to give diphenylethane. It therefore seemed probable that $2: 2^{\prime}$-di-(bromomethyl)diphenyl (II) would react with lithium phenyl to give the mono-lithium compound (III), and that intramolecular reaction between. $\mathrm{CH}_{2} \mathrm{Br}$ and. $\mathrm{CH}_{2} \mathrm{Li}$ would be quicker than interaction between the same groups in two different molecules. This proved to be true, the reaction being complete in a matter of seconds and the yield of dihydrophenanthrene being 80 per cent.

Kenner and Turner ${ }^{3}$ obtained impure dihydrophenanthrene in poor yield by the action of sodium

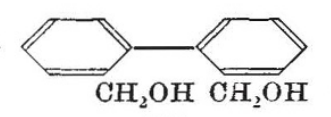

(VI)

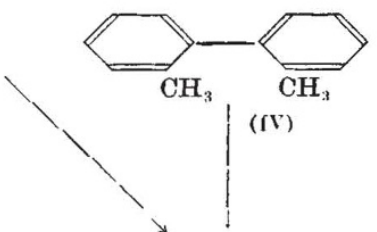

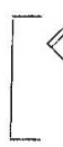

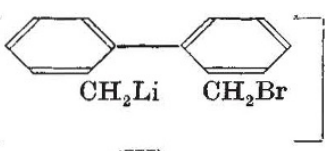

(III)

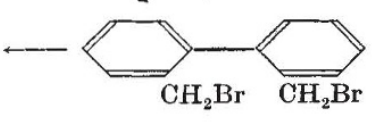

(II) sulphur compounds was observed; metallic sulphates, sulphides and sulphonates appeared to be unreactive, whereas sulphites were slowly reduced and yielded hydrogen sulphide after a period of heating. Thiourea yielded hydrogen sulphide. Presumably any organic sulphur compound which decomposes on heating would respond.

The test promises to be of value in the examination of pigmented materials, dyestuffs and carbonaceous matter, and although it has obvious limitations may prove useful in qualitative analysis as a confirmatory test for free sulphur or as an aid in the identification of organic sulphur compounds.

From preliminary experiments, it would appear that the conversion of sulphur to hydrogen sulphide with polyhydric phenols is quantitative, and that the reaction can be made the basis of a method for estimating sulphur. Work has been carried out in this direction which it is hoped to report later.

Thanks are accorded the Chief Scientist, Ministry of Supply, for permission to publish this note.

A. H. Pettinger

Chemical Inspectorate,

Ministry of Supply. Sept. 30 .

\section{A New Route to Dihydrophenanthrene}

WE are at present engaged in the investigation of the configurations of certain reduced aromatic systems, and have been surprised to find how relatively inaccessible is $9: 10$-dihydrophenanthrene (I). Quite apart from the appreciable difficulty of obtaining pure phenanthrene, the reduction of this hydro- on $2: 2^{\prime}$-di-(bromomethyl)diphenyl, which they made by brominating $2: 2^{\prime}$-ditolyl (IV), formed by an Ullmann reaction on $o$-iodotoluene. We find that the ditolyl can be obtained in $\mathbf{4 0}$ per cent yield by the Krizewsky-Turner ${ }^{4}$ reaction (cupric chloride on $o$-tolyl magnesium bromide) and that Kenner's bromination conditions cannot be greatly improved.

We have also now found that dimethyl diphenate (V) is quantitatively reduced by ethereal lithium aluminium hydride ${ }^{5}$ in a few minutes to the hitherto unknown 2:2'-di-(hydroxymethyl)-diphenyl (VI), m.p. 112-113 (found: C, 77.9; $\mathrm{H}, 6.8 ; \mathrm{C}_{14} \mathrm{H}_{14} \mathrm{O}_{2}$ requires $\mathrm{C}, 78.5 ; \mathrm{H}, 6.8$ per cent), and that this alcohol reacts smoothly and rapidly with aqueous hydrobromic acid at about $100^{\circ}$ to give $2: 2^{\prime}$-di(bromomethyl)diphenyl. Combining the three stages, it is thus possible in little more than a day's work to convert dimethyl diphenate, a readily available substance, into dihydrophenanthrene in an overall yield of about 70 per cent. We are investigating the general applicability of the method.

D. Muriel Hall E. E. TURNER

Department of Chemistry, Bedford College, University of London. Oct. 8.

1 Burger, A., and Mosettig, E., J. Amer. Chem. Soc. 57, 2731 (1935): 58, 1857 (1936). Cf. Durland, J. R., and Adkins, H., ibid., ! 9 . 135 (1937).

'Wittig, G., and Pockels, U., Ber., 72, 884 (1939). Wittig, G., and Witt, H., Ber., 74, 1474 '(1941).

4 Krizewsky, J., and Turner, E. E., J. Chem. Soc., 115, 559 (1919). Turner, E. E., J. and Proc. Roy. Soc. N.S. Wales, 54, 37 (1920)

${ }^{\circ}$ Finholt, A. E., Bond, A. C., jun., and Schlesinger, H. I., J. Amer. Chem. Soc, 69, 1199 (1947). Nystrom, R. F., and Brown, W. G., ibid., 69, 1197, 2548 (1947).
${ }^{3}$ Kenner, J., and Turner, E. G., J. Chem. Soc., 99, 2101 (1911). 